\title{
Frecuencia de malignidad en nódulos pulmonares en adultos
}

Dr. José Rodrigo Hernández Gutiérrez

\section{Resumen}

En cuanto a los antecedentes el cáncer de pulmón es la segunda causa más común de cáncer en el mundo, tanto en los hombres como en las mujeres. La presentación inicial de cáncer pulmonar es en forma de nódulos. El objetivo fue determinar la frecuencia de malignidad en nódulos pulmonares de la población adulta. El diseño fue descriptivo, transversal. Se realizó en la unidad de cirugía de tórax del Instituto Guatemalteco de Seguridad Social (IGSS). En cuanto a los materiales y métodos utilizados consistió en revisión de expedientes de pacientes sometidos a cirugía por presentar nódulo pulmonar, en el periodo de 2000 a 2016. Las frecuencias de malignidad se realizaron por porcentajes con intervalo de confianza de $90 \%$. Las asociaciones de variables por medio de chi2 y odds ratio $(p<0.1)$. Se estableció que, de los 77 pacientes sometidos a cirugía por nódulos pulmonares, 36 con hallazgo de malignidad. El paciente con antecedente de cáncer presenta 4 veces más riesgo de malignidad. La ausencia de calcificación en el nódulo aumenta 55 veces más el riesgo de malignidad de nódulos pulmonares. Con lo que se llegó a la concluir que la frecuencia de malignidad en nódulos pulmonares de la población sometida a cirugía es de 34-60 \% (IC 90 \%). La población con antecedente de cáncer personal tiene cuatro veces mayor riesgo de malignidad en la presentación de nódulos pulmonares. La presencia de calcificación en nódulos se considera como factor protector frente la malignidad.

Palabras clave: nódulo pulmonar solitario, múltiples nódulos pulmonares, malignidad. 


\title{
Frequency of malignancy in pulmonary nodes of adult patients
}

\author{
Abstract
}

As already known, lung cancer is the second most common cause of cancer in the world, in both men and women. The initial signs of lung cancer are nodes or nodules. The purpose of this study was to determine the frequency of malignancy in the pulmonary nodes of adult populations. Its design was descriptive and cross-sectional. It was performed at the Chest Surgery Unit of the Guatemalan Institute of Social Security (Instituto Guatemalteco de Seguridad Social, IGSS). In regards to materials and methodology, the study reviewed the medical files of patients who had undergone surgery due to presence of pulmonary nodes during the period of 2000-2016. The malignancy rates were estimated with a $90 \%$ confidence interval, variable associations by chi2 and an odds ratio $(p<0.1)$. It was established that in the sample of 77 patients who had surgery for pulmonary nodes, 36 were found to be malignant. The patient with a history of cancer has four times more risk of malignancy. The absence of node calcification increases the risk of pulmonary node malignancy by 55 times. Thus, the study concluded that frequency of pulmonary node malignancy is 34-60\% (Cl $90 \%)$ in populations who undergo surgery. People with a personal history of cancer have four times more risk of pulmonary node malignancy. The existence of node calcification is considered a protecting factor against malignancy.

Key words: solitary pulmonary node, multiple pulmonary nodes, malignancy. 


\section{Introducción}

El cáncer de pulmón es la segunda causa más común de cáncer en el mundo, tanto en los hombres como en las mujeres. En los hombres es superado solamente por cáncer de próstata y en las mujeres por el cáncer de mama (1). Los nódulos pulmonares son lesiones que pueden evidenciarse en una radiografía de tórax como un hallazgo incidental o dirigido. Estas lesiones pueden ser únicas o pueden ser múltiples. Se debe de tener en cuenta que los nódulos pulmonares son comunes, sobre todo, en personas mayores de 50 años. El problema es determinar cuál de esos nódulos pueden ser benignos y cuáles son un preámbulo para enfermedad cancerosa o ya ser cáncer instaurado. Se debe de tener en cuenta que todo cáncer pulmonar inicia con presencia de uno o varios nódulos pulmonares, pero no todos los nódulos pulmonares son cáncer. Cuando se habla de nódulo pulmonar, se refiere a una lesión mayor a 5 milímetros y menor o igual de 3 centímetros. Una lesión mayor de $3 \mathrm{~cm}$, ya es considerada como masa, la cual tiene un mayor riesgo de ser maligna. Las causas de nódulos pulmonares van desde enfermedad maligna primaria extra torácica, hasta una infección (2), (3), (4), (5), (6).

\section{Materiales y métodos}

El presente es un estudio transversal descriptivo, donde se revisan 77 expedientes de población adulta sometida a cirugía, para toma de biopsia excisión, por la aparición de nódulo pulmonar solitario o múltiples nódulos pulmonares.
Se diseñó una boleta de recolección de datos. En la cual se llenó con la información que se obtuvo de la revisión de expedientes. La boleta se divide en tres incisos: el primero con datos generales del paciente: nombre, número de afiliación, sexo y edad. El segundo con antecedentes del paciente: tabaquismo e historia de cáncer. El tercer inciso de estudios complementarios: informe de radiología y número de esta, así como informe de biopsia y su numeración correspondiente.

Los expedientes de los pacientes contienen la información recolectada, desde los datos generales hasta el informe radiológico de la tomografía que se les realizó, también resultado final patológico.

La información registrada en la boleta de recolección de datos fue almacenada en una base de datos, en una plantilla de Microsoft Excel, para su posterior análisis estadístico. Se realiza determinación de frecuencias, expresadas en porcentajes con intervalos de confianza de $90 \%$. Luego se realizan medidas de asociación en las que se utiliza la prueba de chi2 junto con odds ratio ( $p$ $<0.1$ ). Luego de determinar las frecuencias individuales de cada variable, se procede a realizar las medidas asociativas, se compara cada variable, contra la frecuencia de malignidad.

Se presentarán los resultados de una manera sintetizada en la tabla 1, que contiene caracterización epidemiológica y en la tabla 2, que contiene la caracterización clínica. 


\section{Resultados}

A continuación, presentación de los resultados.

Tabla 1

Caracterización epidemiológica de los pacientes, $\mathrm{n}=77$ pacientes

\begin{tabular}{|c|c|c|c|}
\hline Características & & $\begin{array}{l}\text { Número de } \\
\text { casos }(\mathrm{n})\end{array}$ & Porcentaje (IC $90 \%$ ) \\
\hline \multirow{2}{*}{ Sexo } & Masculino & 35 & $45 \%(32,59)$ \\
\hline & Femenino & 42 & $55 \%(41,68)$ \\
\hline \multirow{2}{*}{ Edad } & 23-50 años & 39 & $51 \%(37,64)$ \\
\hline & $51-83$ años & 38 & $49 \%(36,63)$ \\
\hline \multirow{2}{*}{ Residencia } & Región metropolitana & 56 & $73 \%(61,84)$ \\
\hline & Otras regiones & 21 & $27 \%(16,39)$ \\
\hline \multirow{2}{*}{$\begin{array}{l}\text { Ambiente } \\
\text { de ocupación }\end{array}$} & Cerrado & 45 & $58 \%(45,71)$ \\
\hline & Abierto & 32 & $42 \%(29,55)$ \\
\hline \multirow{2}{*}{$\begin{array}{l}\text { Antecedente de } \\
\text { cáncer familiar }\end{array}$} & Sí & 6 & $8 \%(6,15)$ \\
\hline & No & 71 & $92 \%(85,99)$ \\
\hline \multirow{2}{*}{$\begin{array}{l}\text { Antecedente de } \\
\text { cáncer personal }\end{array}$} & Sí & 35 & $45 \%(32,59)$ \\
\hline & No & 42 & $55 \%(41,68)$ \\
\hline \multirow{2}{*}{$\begin{array}{l}\text { Antecedente } \\
\text { de tabaquismo }\end{array}$} & Sí & 25 & $32 \%(29,55)$ \\
\hline & No & 52 & $68 \%(55,80)$ \\
\hline \multirow{2}{*}{$\begin{array}{l}\text { Antecedente } \\
\text { de tuberculosis }\end{array}$} & Sí & 3 & $4 \%(0.1,11)$ \\
\hline & No & 74 & $96 \%(91,101)$ \\
\hline \multirow{2}{*}{ Paciente VIH positivo } & Sí & 0 & $0 \%(-)$ \\
\hline & No & 77 & $100 \%(-)$ \\
\hline
\end{tabular}

Fuente: elaboración propia con base de datos obtenidos de Departamendo de Cirugía de Tórax del Instituto Guatemalteco de Seguridad Social. 
Tabla 2

Caracterización clínica de pacientes, $n=77$ pacientes

\begin{tabular}{|c|c|c|c|}
\hline Características & & $\begin{array}{l}\text { Número de } \\
\text { casos (n) }\end{array}$ & Porcentaje (IC $90 \%$ ) \\
\hline \multirow{2}{*}{$\begin{array}{l}\text { Cantidad } \\
\text { de nódulos }\end{array}$} & Nódulo pulmonar solitario & 41 & $53 \%(40,60)$ \\
\hline & Múltiples nódulos pulmonares & 36 & $47 \%(34,60)$ \\
\hline \multirow{3}{*}{$\begin{array}{l}\text { Localización } \\
\text { de pulmón }\end{array}$} & Izquierdo & 20 & $26 \%(11,41)$ \\
\hline & Derecho & 32 & $42 \%(26,57)$ \\
\hline & Ambos & 25 & $32 \%(17,48)$ \\
\hline \multirow[t]{2}{*}{ Tamaño } & $5 \mathrm{~mm}-15 \mathrm{~mm}$ & 36 & $47 \%(34,60)$ \\
\hline & $16 \mathrm{~mm}-30 \mathrm{~mm}$ & 41 & $53 \%(40,66)$ \\
\hline \multirow[t]{2}{*}{ Calcificación } & Sí & 26 & $34 \%(21,46)$ \\
\hline & No & 51 & $66 \%(54,79)$ \\
\hline \multirow[t]{2}{*}{ Bordes } & Bien definidos & 46 & $60 \%(47,73)$ \\
\hline & Irregulares & 31 & $40 \%(27,53)$ \\
\hline \multirow[t]{2}{*}{ Hallazgo } & Maligno & 36 & $47 \%(34,60)$ \\
\hline & Benigno & 41 & $53 \%(40,66)$ \\
\hline
\end{tabular}

Fuente: elaboración propia con base de datos obtenidos de Departamendo de Cirugía de Tórax del Instituto Guatemalteco de Seguridad Social.

La información mostrada en los cuadros, se generó a partir del trabajo de campo. Se presentan los porcentajes de las frecuencias encontradas, acompañadas de su intervalo de confianza de $90 \%$. Los resultados mostrados en las tablas 1 y 2 muestran frecuencias de cada variable, con intervalos de confianza calculados al $90 \%$.

En la tabla 1 se consideraron las variables de sexo, edad, ambiente de ocupación y antecedente personal de cáncer; presentaron frecuencias muy parecidas entre ellas. No mostraron una diferencia muy marcada en sus porcentajes.

Las variables de lugar de residencia, antecedente familiar de cáncer, antecedente de tabaquismo, VIH positivo y antecedente de tuberculosis. Tuvieron una marcada diferencia. Predominó las personas residentes en la región metropolitana, mientras que el mayor porcentaje de las otras variables mencionadas es negativo.

En la tabla 2 se consideraron las variables clínicas. Indicaron que no hay una frecuencia que favorezca marcadamente al nódulo pulmonar solitario ni a los múltiples nódulos pulmonares. La localización pulmonar, como el tamaño no tienen una frecuencia marcada. El porcentaje de malignidad es menor al de benignidad, pero las frecuencias no se encuentran muy separadas. 
Frecuencia de malignidad en nódulos pulmonares, $n=77$ pacientes

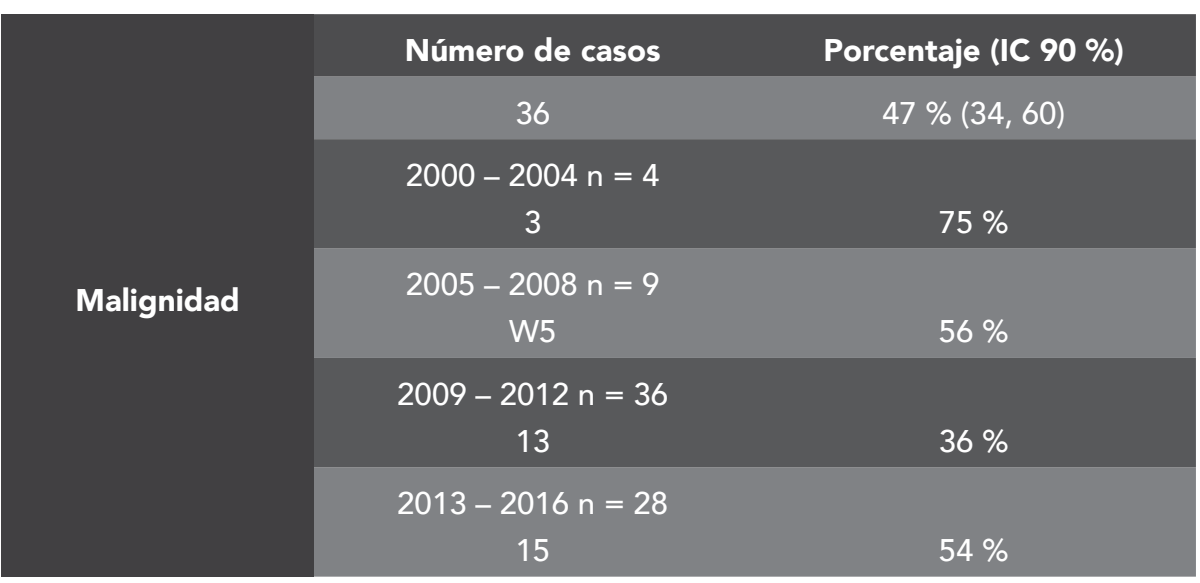

Fuente: elaboración propia con base en datos obtenidos de Departamendo de Cirugía de Tórax del Instituto Guatemalteco de Seguridad Social.

En la tabla 3, se muestra la frecuencia de malignidad de las personas que fueron sometidas a cirugía luego de presentar uno o múltiples nódulos pulmonares. Se evidencia que el $53 \%$ de la población del estudio presentaron patología no maligna, mientras que el $47 \%$ restante mostraron patología maligna. Del $47 \%$ de patología maligna, el $16 \%$ presentó cáncer pulmonar primario y $31 \%$ de cáncer metastásico.

\section{Discusión}

La frecuencia de malignidad en la población adulta, del Instituto Guatemalteco de Seguridad Social, sometida a cirugía por presentar nódulos pulmonares es de $34-60 \%$. Valor muy importante, basándose en los resultados de Bai $C_{\text {, et al. En }}$ Asia, en la evaluación de los nódulos pulmonares, el grupo de Bai C, reporta malignidad de $5 \%$ de la población con presencia de nódulos (3).

Se debe mencionar, la población del estudio en el IGSS es muy pequeña. Es importante indicar, la falta de estudios de nódulos pulmonares en Guatemala.

Esto se menciona por el incumplimiento mínimo que conlleva la presencia de nódulos pulmonares. El cumplimiento mínimo de abordaje debe ser la tomografía de tórax y toma de biopsia (4). Existe la posibilidad que muchos pacientes con presencia de nódulos pulmonares en Guatemala no están siendo estudiados.

Luego de realizar las medidas de asociación indicadas previamente, las cuales incluyen: chi2 de Pearson y odds ratio, utilizando un valor de $p<0.10$. Se discutirá de manera profunda, las variables significativas y se hará mención las variables con poco valor estadístico.

Las variables de sexo, lugar de residencia, ocupación que desarrollan, antecedente de cáncer familiar, antecedente de tabaquismo, $\mathrm{VIH}$ positivo, antecedente de tuberculosis, tamaño del nódulo (en caso de múltiples nódulos, se tomó en cuenta el más grande) y bordes del nódulo (en caso de múltiples, se tomó en cuenta el más grande). Luego de ser sometidas en la prueba de chi2, asociándolas con la malignidad de los nódulos pulmonares, con un valor de $p<0.1$, no tienen valor significativo en la asociación. Por lo que se puede generar el argumento que ninguna de estas variables está asociada con la frecuencia de la malignidad, en la población estudiada.

Existen dos variables muy bien descritas en el riesgo de malignidad, las cuales son: edad de presentación y cantidad de nódulos.

En la edad de presentación Mandel y Stark (4), en Estados Unidos, indican que la población menor de 50 años que presentan un porcentaje de malignidad de 3-15\%; mientras que la población de este estudio posee un porcentaje de 37-64 \%. La población mayor de 50 años, de los estadounidenses, el porcentaje de malignidad es de 43-93\%; cuando en este estudio el porcentaje de malignidad en esa población es de 36-63\%. Claramente los resultados no 
son parecidos, hay diferencia muy marcada, afectó mucho la cantidad de pacientes evaluados.

En la cantidad de nódulos pulmonares Weinberger y McDermontt (7), describen que tiene mayor malignidad el nódulo pulmonar solitario, sobre la presencia de múltiples nódulos. En el estudio luego de someter a chi2 la variable de cantidad de nódulos, asociándola a malignidad se evidenció un resultado de 2.1, valor por debajo del valor esperado de 2.71. Por lo que, en este estudio, la cantidad de nódulos pulmonares no es un marcador confiable para la frecuencia de la malignidad.
Las variables con un gran valor estadístico fueron: la calcificación de nódulos y el antecedente de cáncer personal. La calcificación se toma como un factor protector. Se realiza la asociación de odds ratio, con un valor de $p<0.1$, lo cual da un resultado de 0.018 . Lo anterior puede interpretarse con un factor protector de la calcificación ante la malignidad. Haciendo la asociación por medio de odds inverso se tiene un resultado de 55. Por lo que se evidencia, la ausencia de calcificación aumenta en 55 veces la posibilidad de malignidad del nódulo.

El antecedente personal de cáncer es determinante al realizar la asociación con la malignidad de nódulos pulmonares. Luego

\title{
Referencias
}

1. American Cancer Society. Cancer prevention \& early detection facts \& figures 2015-2016. Atlanta: American Cancer Society; 2015. Disponible en: https:// www.cancer.org/research/cancer-facts-statistics/cancer-prevention-earlydetection.html

2. Lung Cancer Alliance. Lung cancer screening: Understanding lung nodules. Washington, DC: Lung Cancer Alliance; 2014. Disponible en: https://oregon. providence.org/ /media/Files/Providence\%20OR\%20Migrated\%20PDFs/ Patients\%20Toolkit/Nodules\%20Brochure\%202014\%20FINAL.pdf

1. Bai C, Yim A et al. Evaluation of pulmonary Nodules Clinical Practice Consensus Guidelines for Asia. Chest. Octubre 2016;4(150):877-893.

2. Thomas KW, Gould MK. Overview of the initial evaluation, diagnosis and staging of patients with suspected lung cancer. In UpToDate, Post TW (Ed), UpToDate, Waltham. MA. Disponible en: https://www.uptodate.com/contents/overviewof-the-initial-evaluation-diagnosis-and-staging-of-patients-with-suspectedlung-cancer?search=differential\%20diagnosis\%20and\%20 evaluation\%20of\%20 multiple\%20pulmonary\%20nodules\&topicRef=6986\&source=see_link

3. Wahidi MM, Govert JA, Goudar RK, Gould MK, McCrory DC. Evidence for the treatment of patients with lung nodules. ACCP Evidence-Based Clinical Practice Guidelines. Chest. Septiembre de 2007;132(3):94S-107S.

4. Callister MEJ, et al. BTS Guidelines for the investigation and management of pulmonary nodules. Thorax an international journal of respiratory medicine. 2015;70(2):ii1-ii54

5. Weinberger SE, McDermontt S. Diagnostic evaluation of the incidental pulmonary nodule. UpToDate. 2020. Disponible en: https://www.uptodate. $\mathrm{com} /$ contents/diagnostic-evaluation-of-the-incidental-pulmonarynodule?search=diagnostic\%20and\%20 valuation\%20 of\%20incidental\%20 pulmonary\%20nodule\&source=search_result\&selectedTitle=1 6\&usage _ type $=$ default\&display_rank=1

de la realización de odds ratio con valor de $p<0.1$, se obtiene un valor de 4.27 . Esto evidencia que las personas que tiene historia de cáncer previo, sin importar el origen, tienen 4 veces mayor riesgo de malignidad en la aparición de nódulos pulmonares, aumenta la frecuencia de malignidad en estos pacientes, pero se debe tener en cuenta que estos pacientes, en el momento de presentar cáncer de cualquier origen, la metástasis siempre será una consecuencia difícil de controlar.

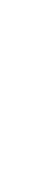

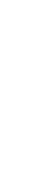

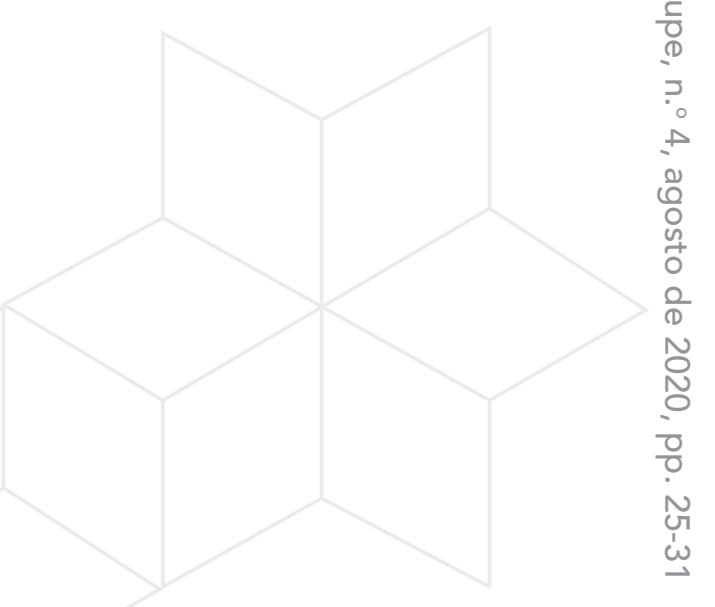

